Article

\title{
Rogue Wave Modes for the Coupled Nonlinear Schrödinger System with Three Components: A Computational Study
}

\author{
Hiu Ning Chan * and Kwok Wing Chow \\ Department of Mechanical Engineering, University of Hong Kong, Pokfulam, Hong Kong, China; \\ kwchow@hku.hk \\ * Correspondence: hnchan06@connect.hku.hk; Tel.: +852-3917-2641; Fax: +852-2858-5415 \\ Academic Editor: Boris Malomed \\ Received: 13 April 2017; Accepted: 16 May 2017; Published: 29 May 2017
}

\begin{abstract}
The system of "integrable" coupled nonlinear Schrödinger equations (Manakov system) with three components in the defocusing regime is considered. Rogue wave solutions exist for a restricted range of group velocity mismatch, and the existence condition correlates precisely with the onset of baseband modulation instability. This assertion is further elucidated numerically by evidence based on the generation of rogue waves by a single mode disturbance with a small frequency. This same computational approach can be adopted to study coupled nonlinear Schrödinger equations for the "non-integrable" regime, where the coefficients of self-phase modulation and cross-phase modulation are different from each other. Starting with a wavy disturbance of a finite frequency corresponding to the large modulation instability growth rate, a breather can be generated. The breather can be symmetric or asymmetric depending on the magnitude of the growth rate. Under the presence of a third mode, rogue wave can exist under a larger group velocity mismatch between the components as compared to the two-component system. Furthermore, the nonlinear coupling can enhance the maximum amplitude of the rogue wave modes and bright four-petal configuration can be observed.
\end{abstract}

Keywords: rogue waves; manakov system; modulation instability; numerical simulation

\section{Introduction}

Rogue waves or freak waves are extreme events in the ocean which are characterized by the emergence of large waves from an otherwise tranquil background [1-3]. The unexpectedly large displacements of the sea surface pose threats to maritime activities and offshore structures. Intensive research efforts are conducted to understand the physics of rogue wave and to develop measures to predict or detect such waves [4,5]. Although rogue waves originate from the context of water waves [1-3,6-9], these studies have been extended to other physical contexts like optical fibers [10-12] and Bose-Einstein condensates [13]. Moreover, it has been demonstrated that optical rogue waves are related to supercontinuum generation $[10,11]$. The high repetition rate of optical experiments is an advantage in the study of these rare events.

For hydrodynamic surface waves, the nonlinear Schrödinger (NLS) equation governs the slow evolution of a weakly nonlinear wave packet [2]. The NLS equation can also describe the dynamics of temporal pulses in an optical fiber [10]. The Peregrine breather of the NLS equation is localized in both space and time, and is a widely utilized model for rogue waves [14,15]. This solution is only nonsingular in the focusing regime unless higher order terms are considered [16].

When there are two or more wave trains present, the governing model is then the system of coupled nonlinear Schrödinger equations $[17,18]$. The special case where all the coefficients of cubic 
nonlinearities are identical is known as the Manakov system. In optics, the Manakov equations provide an analytical account on the propagation of two optical beams in a photorefractive medium [19]. Moreover, when polarization effects are taken into account, the evolution of slowly varying, mutually perpendicular electric fields along an optical fiber with birefringence can be described by a system of coupled NLS equations too [20]. Another relevant example in optical physics is the issue of data communication using multi-mode fibers. Spatial multiplexing can relieve the capacity constraints of single-mode fibers. Random coupling of degenerate or quasi-degenerate modes for multi-mode fibers in the nonlinear propagation regime can then lead to generalized Manakov equations with many components [21]. Subsequently, transmissions in multi-mode fibers exhibiting rapidly varying birefringence will also give rise to Manakov equations upon averaging [22], and intermodal modulation instabilities for coupled Schrödinger systems can be analyzed [23]. Finally, the Manakov system is also a useful model in the dynamics of cold atomic species studied in Bose-Einstein condensates [13,24].

Theoretically, the Manakov equations are obtained by considering the evolution of slowly varying electric fields in temporal or spatial waveguides. For temporal waveguides, group velocity dispersion is balanced by Kerr (cubic) nonlinearity. Rogue waves of the two-component Manakov system in the focusing regime have been studied intensively [25-27]. In contrast to the single component case, rogue wave modes had been discovered for the coupled NLS equations in the defocusing regime [28-31] with group velocity mismatch between the two components. This scenario is closely related to new ranges of modulation instability in the defocusing regime. Theoretically, baseband instability is associated with the unstable behavior of disturbances in the low frequency regime. Remarkably these theoretical studies are amply supported by experimental efforts. Indeed, wavelength-division-multiplexed systems have been extended in the laboratory setting beyond the soliton formation regime, or more precisely to baseband and passband regimes for polarization modulation instabilities and the existence of rogue wave modes of the Manakov equations [32]. In another multi-component investigation, optical dark rogue waves are demonstrated by a suitable injection of two colliding and modulated pump beams with orthogonal states of polarization [33]. Furthermore, rogue waves for the three-component Manakov system in the focusing regime had been derived. Four-petal patterns are possible [34,35]. Such wave profiles are otherwise inadmissible for the one-component and two-component counterparts. Interactions of rogue waves with solitons and breathers were investigated by utilizing the Darboux transformation [36].

In this work, we extend the study to the three-component Manakov system in the defocusing regime. For mathematical convenience, the system is taken in the non-dimensional and normalized form. We incorporate symmetrically placed group velocity differences between the complex valued components $u, v$, and $w$ :

$$
\begin{aligned}
& i u_{z}+i \delta u_{t}+u_{t t}-\sigma\left(|u|^{2}+|v|^{2}+|w|^{2}\right) u=0 \\
& i v_{z}-i \delta v_{t}+v_{t t}-\sigma\left(|u|^{2}+|v|^{2}+|w|^{2}\right) v=0 \\
& i w_{z}+w_{t t}-\sigma\left(|u|^{2}+|v|^{2}+|w|^{2}\right) w=0
\end{aligned}
$$

where $\delta$ describes the group velocity mismatch and $\sigma$ measures the coefficient of cubic nonlinearity.

The objective of this paper is to investigate the dynamics of rogue waves and breathers in a multi-component system through a combination of theoretical perspective and computational approach. Theoretically, the formation of rogue wave and breathers can be explained in terms of modulation instability (MI). A detailed numerical investigation on the evolution of a plane wave perturbed by a single wavy disturbance would supplement the theoretical framework. In particular, a disturbance from the baseband of the MI spectrum would generate a rogue wave. For a mode with a finite frequency, both symmetric and asymmetric breathers are observed from the computational study.

The outline of the paper is as follows. In Section 2, the rogue wave mode is derived by the Hirota bilinear method. The coupling effect is discussed in Section 3. The generation of rogue wave mode from baseband modulation instability is confirmed directly by computer simulation 
in Section 4. Similarly, the generation of symmetric and asymmetric breathers is demonstrated in Section 5. In Section 6, a numerical method for finding rogue wave modes in non-integrable systems is proposed. The conclusion is drawn in Section 7.

\section{Formulation of the Rogue Wave Modes}

Under the transformations, $u=\rho_{1} \exp \left(-i \omega_{1} z\right) \frac{g_{1}}{f}, v=\rho_{2} \exp \left(-i \omega_{2} z\right) \frac{g_{2}}{f}, w=\rho_{3} \exp \left(-i \omega_{3} z\right) \frac{g_{3}}{f}$, Equation (1) can be rewritten in terms of the Hirota bilinear operator [37] as

$$
\begin{gathered}
{\left[i D_{z}+i \delta D_{t}+D_{t}^{2}\right] g_{1} \cdot f=0,} \\
{\left[i D_{z}-i \delta D_{t}+D_{t}^{2}\right] g_{2} \cdot f=0,} \\
\left(i D_{z}+D_{t}^{2}\right) g_{3} \cdot f=0, \\
\left(D_{t}^{2}-C\right) f \cdot f=-\sigma\left(\rho_{1}^{2}\left|g_{1}\right|^{2}+\rho_{2}^{2}\left|g_{2}\right|^{2}+\rho_{3}^{2}\left|g_{3}\right|^{2}\right),
\end{gathered}
$$

where $\omega_{1}=\omega_{2}=\omega_{3}=C$, and $C=\sigma\left(\rho_{1}^{2}+\rho_{2}^{2}+\rho_{3}^{2}\right)$.

The methodology in deriving the rogue wave solution is similar to that used in our earlier works [31] and thus the details are omitted here. The amplitude of the plane wave background, $\varrho$, is taken to be identical for all three waveguides. Basically a breather is first derived using a two-soliton expression with complex conjugate wavenumbers. By taking the small frequency limit of the breather, the rogue wave mode is given by

$$
\begin{gathered}
u=\rho \exp \left(-3 i \sigma \rho^{2} z\right)\left\{1-4 \frac{1+i(b-\delta) t+i\left(a^{2}-b^{2}+b \delta\right) z}{\left[a^{2}+(b-\delta)^{2}\right]\left[(t-b z)^{2}+a^{2} z^{2}+\frac{1}{a^{2}}\right]}\right\}, \\
v=\rho \exp \left(-3 i \sigma \rho^{2} z\right)\left\{1-4 \frac{1+i(b+\delta) t+i\left(a^{2}-b^{2}-b \delta\right) z}{\left[a^{2}+(b+\delta)^{2}\right]\left[(t-b z)^{2}+a^{2} z^{2}+\frac{1}{a^{2}}\right]}\right\}, \\
w=\rho \exp \left(-3 i \sigma \rho^{2} z\right)\left\{1-4 \frac{1+i b t+i\left(a^{2}-b^{2}\right) z}{\left(a^{2}+b^{2}\right)\left[(t-b z)^{2}+a^{2} z^{2}+\frac{1}{a^{2}}\right]}\right\},
\end{gathered}
$$

where $a$ and $b$ are the real part and imaginary part of $\Omega_{0}: \Omega_{0}=a+i b$. The parameter $\Omega_{0}$ is the leading order term in the asymptotic expansion of the wavenumber, which satisfies the dispersion relation,

$$
\Omega_{0}^{6}+2\left(\delta^{2}+3 \sigma \rho^{2}\right) \Omega_{0}^{4}+\delta^{4} \Omega_{0}^{2}+2 \delta^{4} \sigma \rho^{2}=0 .
$$

This cubic polynomial in $\Omega_{0}^{2}$ will dictate the dynamics and profiles of the rogue waves, to be highlighted in the following section. 


\section{The Effect of Coupling}

\subsection{Extension of Existence Regime}

The rogue wave solution in Equation (2) is nonsingular if and only if $a$ never vanishes. Since $\Omega_{0}$ cannot be real, this is equivalent to having a non-real root for the cubic polynomial $p_{3}(x)=x^{3}+2\left(\delta^{2}+3 \sigma \rho^{2}\right) x^{2}+\delta^{4} x+2 \delta^{4} \sigma \rho^{2}$. By considering the discriminant of $p_{3}(x)$, complex roots (and hence rogue waves) will exist for $0<\delta^{2}<16.9 \sigma \rho^{2}$. One highlight of the present work is that this constraint is much less restrictive than the corresponding existence condition for the Manakov system with two components, namely, $0<\delta^{2}<4 \sigma \rho^{2}$ [31]. We conjecture that the addition of more components to coupled systems may in general induce further modulation instabilities and enhance the existence of rogue waves. This hypothesis obviously must be tested for other dynamical systems in the future.

Moreover, the incorporation of the third component increases the complexity of the geometry of the wave profiles. Analytically, the dispersion relation is expressed as a higher order algebraic polynomial and will allow multiple rogue wave solutions under the same input physical parameters. From Equation (3), if $a+i b$ is a root of the dispersion relation, then $a-i b$ will also be admissible and provides another rogue wave solution. This phenomenon was also observed in other multi-component system [38-41]. Such multi-rogue-wave scenarios are not allowed in the two-component Manakov system.

\subsection{Enhancement of Amplitude}

For the two-component Manakov system in the defocusing regime [31], the range of amplitude and configurations of the rogue waves in the two components are identical. Either both wave profiles are eye-shaped dark rogue waves (EDRW) with the main displacement below the background, or both patterns are four-petal-shaped rogue waves (FPRW) with two local maxima and two local minima. Moreover, the maximum and minimum values attained are identical in both components.

For the three components case, the scenarios are drastically different as the various components in Equation (1) can exhibit distinct forms of rogue waves and the maximum displacements can be different (Figure 1). Interestingly, there must be at least one component in the form of an EDRW where the minimum amplitude is bounded below by about 0.4 (See Proof in Appendix A). However, the rogue wave solution cannot take the form of an eye-shaped bright rogue wave with the main displacement above the background (See Proof in Appendix A). As compared to the two-component system, the nonlinear coupling of the third component can enhance the rogue wave in two ways: increasing the maximum amplitude and 'squeezing' a bright rogue wave.

The maximum amplitude can be greater than $\sqrt{2} \rho$ (Figure 1a), which is the upper bound of amplitude for the two-component Manakov system [31]. Similar increment in amplitude due to coupling was also found in other coupled systems such as the long wave-short wave resonance model with two short wave components [41] and a system of coupled derivative nonlinear Schrödinger equations [38].

Although the formation of eye-shaped bright rogue wave is also prohibited [31], a tendency towards the formation of bright rogue wave can be observed in the three-component system. Such bright type rogue wave has a four-petal configuration where the saddle point is above the background and is closer to the maximum than the minimum. The $u$-component in Figure 1a exhibits a bright four-petal configuration where the amplitude at the saddle point is about 1.3 and the rogue wave ranges from 0 to about 1.5 . Such geometry closely resembles the widely studied eye-shaped bright rogue wave. 


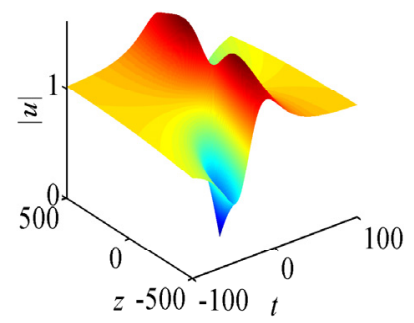

(a)

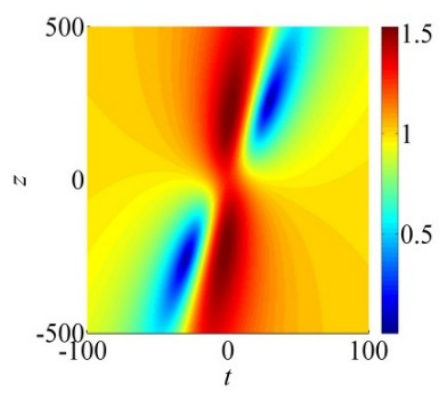

(d)

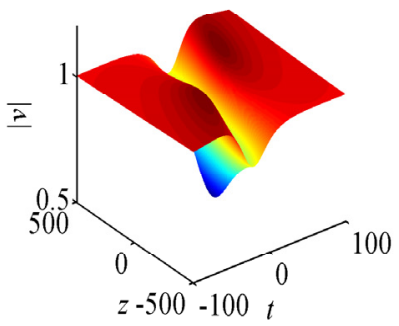

(b)

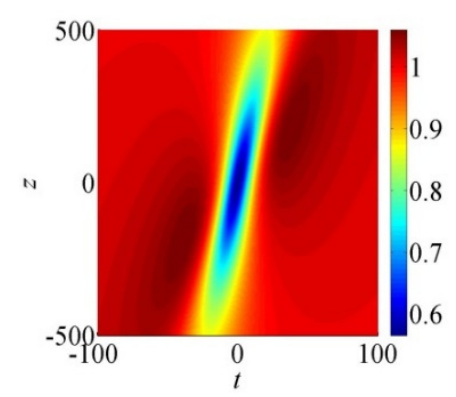

(e)

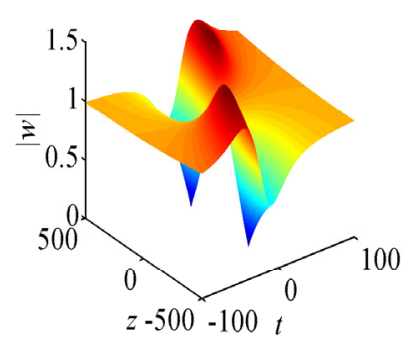

(c)

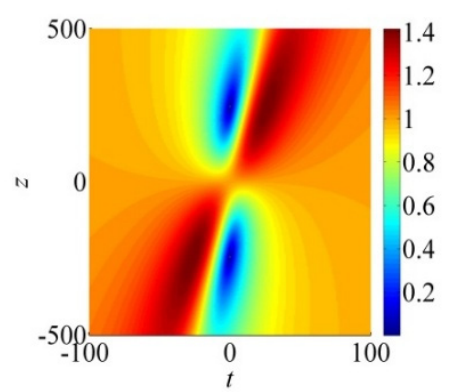

(f)

Figure 1. The rogue wave solution given by Equations (2) and (3) with $\varrho=1, \sigma=1, \delta=0.1$ and $\Omega_{0}=0.0537+0.0537 i$ : (a) $u$-component (bright FPRW); (b) $v$-component (EDRW); (c) $w$-component (FPRW); (d) the top view of (a); (e) the top view of (b); (f) the top view of (c).

\section{Baseband Modulation Instability}

Baseband modulation instability, the instability due to low frequency disturbances, has been shown to be intimately related to the existence condition of rogue waves [29]. The connection was established theoretically in several dynamical systems [38,39,41,42]. This section focuses on the role of baseband modulation instability in the formation of rogue wave.

\subsection{Analytical Approach}

To study the correlation between rogue waves and modulation instability, plane waves with identical amplitude are considered,

$$
\begin{aligned}
u_{0} & =\rho \exp \left[i\left(-3 \sigma \rho^{2} z\right)\right], \\
v_{0} & =\rho \exp \left[i\left(-3 \sigma \rho^{2} z\right)\right], \\
w_{0} & =\rho \exp \left[i\left(-3 \sigma \rho^{2} z\right)\right] .
\end{aligned}
$$

Small perturbations of the form $\exp [i(K t-W z)]$ would be governed by

$$
\begin{aligned}
& W^{6}-\left[3 K^{4}+2\left(\delta^{2}+3 \sigma \rho^{2}\right) K^{2}\right] W^{4}+\left(3 K^{8}+12 \sigma \rho^{2} K^{6}+\delta^{4} K^{4}\right) W^{2} \\
& -\left[K^{12}+2\left(-\delta^{2}+3 \sigma \rho^{2}\right) K^{10}+\delta^{2}\left(\delta^{2}-8 \sigma \rho^{2}\right) K^{8}+2 \sigma \rho^{2} \delta^{4} K^{6}\right]=0
\end{aligned}
$$

Focusing on the instability of low frequency disturbances $(K \rightarrow 0), c=W / K=O(1)$ is determined from $c^{6}-2\left(\delta^{2}+3 \sigma \rho^{2}\right) c^{4}+\delta^{4} c^{2}-2 \sigma \rho^{2} \delta^{4}=0$.

This is identical to the dispersion relation Equation (3) with a slight change in variable: $\left(i \Omega_{0}\right)^{6}-2\left(\delta^{2}+3 \sigma \rho^{2}\right)\left(i \Omega_{0}\right)^{4}+\delta^{4}\left(i \Omega_{0}\right)^{2}-2 \delta^{4} \sigma \rho^{2}=0$, confirming again the relation between baseband modulation instability and rogue waves. 


\subsection{Computational Approach}

The generation of localized modes resembling rogue waves starting from a chaotic field initial condition was studied in the literature [14]. Such modes can only be generated in parameter regimes with baseband modulation instability [42]. Here we demonstrate the emergence of rogue waves from a plane wave perturbed by one single mode instead of a random noise. More precisely, we consider the initial condition

$$
\begin{aligned}
u(t, 0) & =[1+0.05 \exp (i K t)] u_{0}(t, 0), \\
v(t, 0) & =[1+0.05 \exp (i K t)] v_{0}(t, 0), \\
w(t, 0) & =[1+0.05 \exp (i K t)] w_{0}(t, 0),
\end{aligned}
$$

where $u_{0}, v_{0}$ and $w_{0}$ are the plane waves given in Equation (4). Equation (1) is numerically solved with a combination of pseudospectral method and a fourth-order Runge-Kutta scheme [43].

The result is illustrated with the typical case of $\sigma=10, \varrho=1$ and $\delta=5$. The modulation instability gain spectra exhibit multiple bands due to the existence of multiple complex roots of Equation (5) (Figure 2). For small frequency $K$, patterns resembling rogue waves are observed (Figure 3, only patterns for $\mathrm{u}$ are shown, as profiles for the other two waveguides are similar). If there exists a pair of rogue wave modes for the same input parameters, the rogue wave with a higher baseband growth rate would dominate the other mode [39]. However, both modes here share the same baseband growth rate because they correspond to a pair of complex conjugate roots of Equation (3). Similar co-existence of rogue waves in a chaotic wave field was also reported earlier in the literature [40].

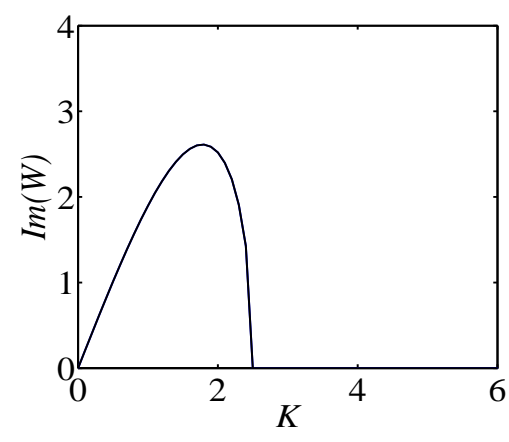

(a)

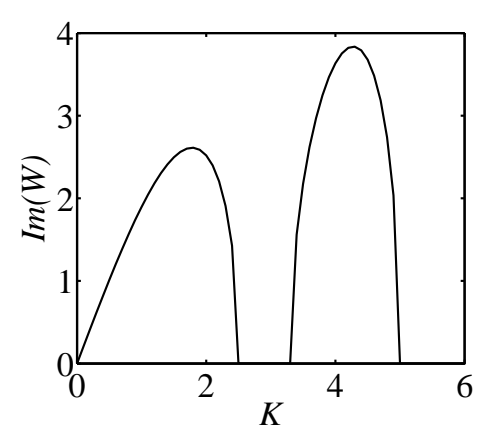

(b)

Figure 2. Multiple bands of the modulation instability gain spectra with $\sigma=10, \varrho=1$ and $\delta=5$ : (a) the first band; (b) the second band.

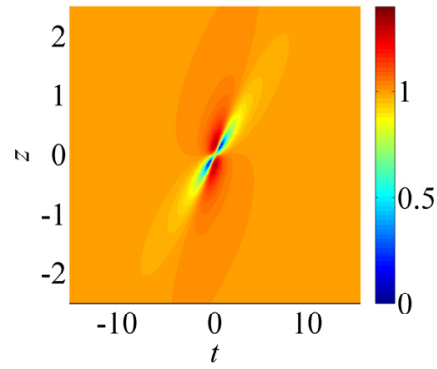

(a)

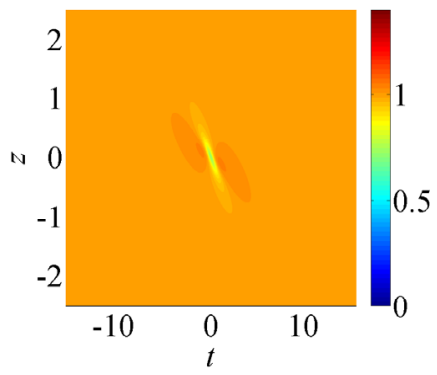

(b)

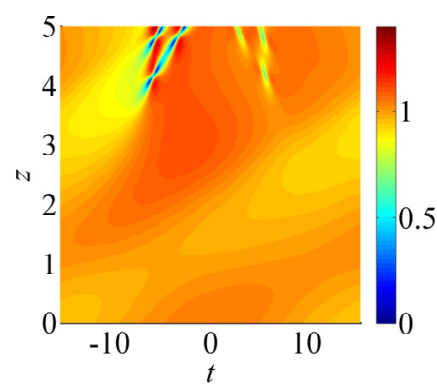

(c)

Figure 3. With $\sigma=10, \varrho=1$ and $\delta=5$, (a) the $u$-component of the first rogue wave mode given by Equations (2) and (3); (b) the $u$-component of the second RW mode; (c) the simulated results with the initial condition given by Equation (6) and $K=0.2$. 


\section{Asymmetric Breathers}

Breathers can be generated through these simulations by starting with a disturbance of higher frequency in the unstable band. For typical values of $\sigma=10, \varrho=1$ and $\delta=5$, there exist two pairs of complex conjugate roots for Equation (5) at the baseband and the instability growth rates are identical. Two breathers can be generated concurrently and superposition leads to an asymmetric breather (Figure 4). For most rogue waves studied in the literature, the local extrema are usually symmetric with respect to the main displacement and attain the same value. For an asymmetric rogue wave or breather [27,44], symmetry is broken, e.g., the four-petal arrangement is destroyed and one of the minimum points splits into two minima (Figure 4a).

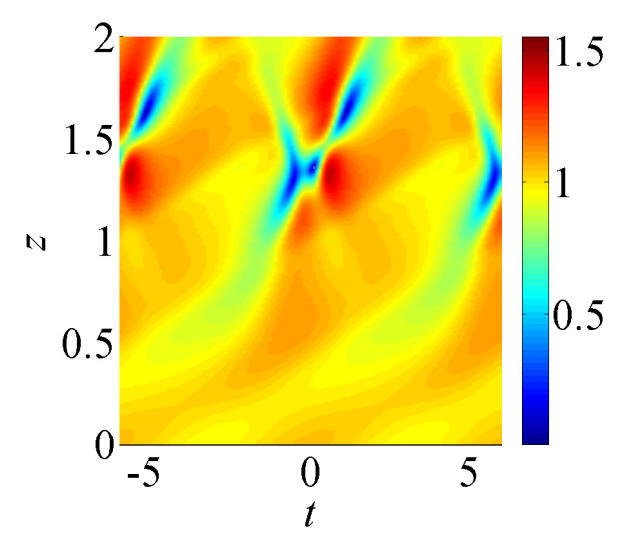

(a)

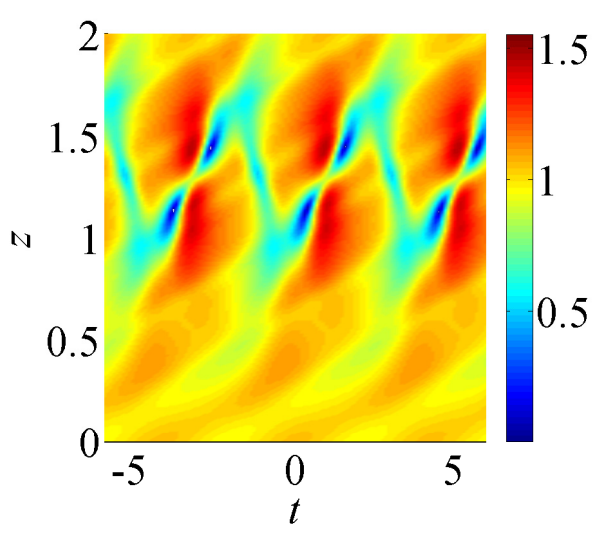

(b)

Figure 4. The $u$-component of the asymmetric breathers generated by simulation with the initial condition given by Equation (6) at (a) $K=1$; (b) $K=1.5$. In both cases, $\sigma=10, \varrho=1$ and $\delta=5$.

\section{Rogue Wave in Non-integrable Systems}

In many physical applications, the coefficients for the self-phase modulation (SPM) and cross-phase modulation (XPM) are distinct from each other, analytical schemes will usually fail for such coupled nonlinear Schrödinger equations $[17,18]$. It will be instructive to apply the mechanism developed here for such 'non-integrable' equations. Based on the numerical solution, wave profile and amplification ratio of the rogue wave can be calculated.

We demonstrate the idea with a special case where the ratio of SPM to XPM is a constant:

$$
\begin{aligned}
& i q_{z}+i \delta^{\prime} q_{t}+q_{t t}-\sigma^{\prime}\left(|q|^{2}+\gamma|r|^{2}+\gamma|s|^{2}\right) q=0, \\
& i r_{z}-i \delta^{\prime} r_{t}+r_{t t}-\sigma^{\prime}\left(\gamma|q|^{2}+|r|^{2}+\gamma|s|^{2}\right) r=0, \\
& i s_{z}+s_{t t}-\sigma^{\prime}\left(\gamma|q|^{2}+\gamma|r|^{2}+|s|^{2}\right) s=0,
\end{aligned}
$$

where $\gamma$ is the ratio of XPM to SPM. Under the initial condition

$$
\begin{aligned}
& q(t, 0)=[1+0.05 \exp (i K t)] \rho, \\
& r(t, 0)=[1+0.05 \exp (i K t)] \rho, \\
& s(t, 0)=[1+0.05 \exp (i K t)] \rho,
\end{aligned}
$$

where $K$ is a low frequency of the wavy disturbance, rogue wave modes are generated (Figures 5 and 6 ). Four-petal RW-like waveforms can be observed with $\gamma=2$ (Figure 5). As compared to Figure 3, the minima get closer and the saddle point attains a smaller intensity. Dark RW-like patterns are observed for the case where XPM is half of SPM (Figure 6). Apparently, only one type of RW waveform can be observed. 


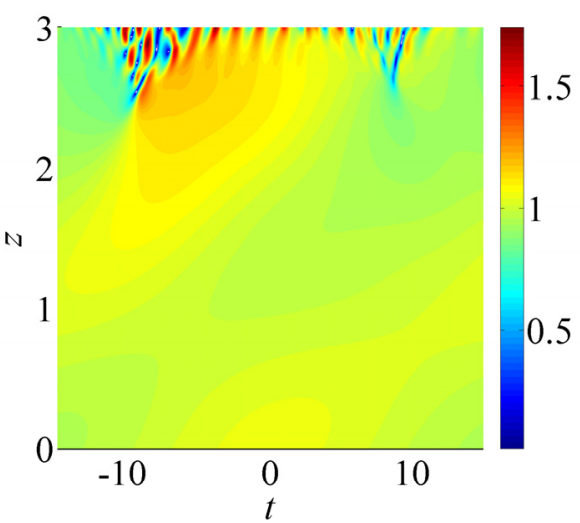

(a)

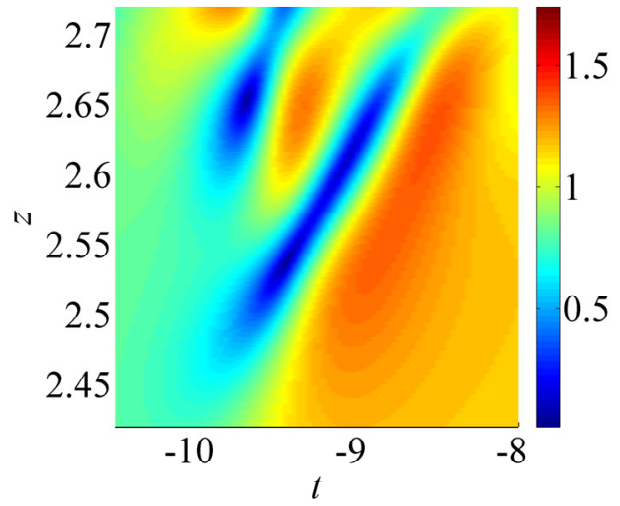

(b)

Figure 5. (a) The $q$-component of the simulated RW mode for Equation (7) with $\sigma^{\prime}=10, \varrho=1, \delta^{\prime}=5$ and $\gamma=2$; (b) an enlarged view of a RW-like structure in (a). The frequency $K$ of the single mode disturbance is 0.2 .

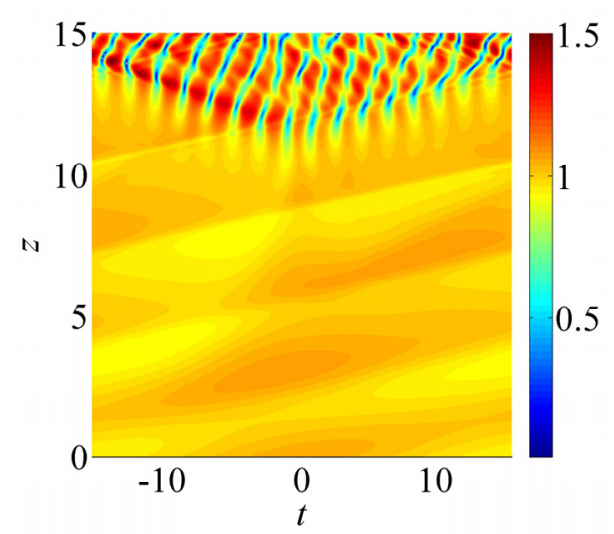

(a)

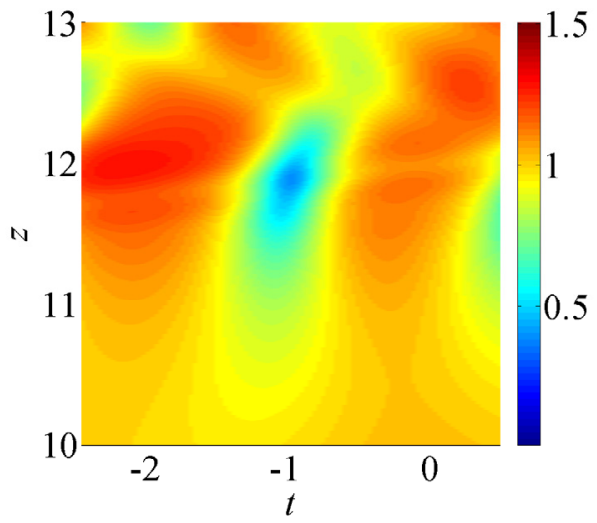

(b)

Figure 6. (a) The $q$-component of the simulated RW mode for Equation (7) with $\sigma^{\prime}=10, \varrho=1, \delta^{\prime}=5$ and $\gamma=0.5 ;(\mathbf{b})$ an enlarged view of a RW-like structure in (a). The frequency $K$ of the single mode disturbance is 0.2 .

In practice, many evolution equations governing dynamical systems for laboratory and engineering settings are not integrable. Several approaches were demonstrated to be feasible as approximations or the estimations of properties of rogue waves in such non-integrable systems. For instance, analytical rogue wave solutions of the NLS equation with periodic modulated coefficients were taken as initial conditions in the numerical approximations of rogue waves for the non-integrable case with constant nonlinearity coefficient and periodic dispersion coefficient [45]. Furthermore, rogue waves from well-studied equations like the NLS equation can be utilized to study rogue waves in less thoroughly studied physical systems. With a suitable physical assumption, optical quadratic solution can be related to the solution of the NLS equation through the second-harmonic asymptotic expansion and the method of repeated substitution [46]. Hence, useful approximations of rogue waves in a quadratic medium can be obtained. It was shown that breathers and RW-like entities emerge from simulations with random initial conditions, which serve as an alternative methodology to examine rogue waves in a general nonlinear system.

In this work, an alternative method is proposed which is based on the generating mechanism of rogue waves. The method is independent of the exact rogue wave solution of the integrable system. Hence, this scheme should hopefully be quite widely applicable to general systems, and should not be restricted to the "quasi-integrable" regime where the coefficients are close to the "integrable" case. As 
compared to the detection of rogue waves in a chaotic field, the baseband disturbance can isolate the rogue-wave-like structures among entities such as breathers. A comprehensive study on rogue waves in a general multi-component system of coupled nonlinear Schrödinger equations will be carried out in the future. This numerical approach initiated from the insight gained from rational solutions should complement the limitation of analytical methods in the study of rogue waves.

\section{Discussions and Conclusions}

It was known that a multi-component Manakov system can effectively model wave propagation in a multicore optical fiber [22]. Besides temporal waveguides, such Manakov systems are relevant in other settings in optical physics too. For spatial solitons, diffraction will play the role of group velocity dispersion, and continuous variations of diffraction, nonlinearity, and gain/loss might lead to novel rogue wave patterns [47]. Similarly, Manakov soliton can arise for biased guest-host photorefractive polymer too [48]. Furthermore, reductive perturbation techniques can be employed to establish Manakov equations as approximations for propagation of electromagnetic fields along isotropic chiral metamaterials [49].

In this work, a theoretical study is performed to understand the increasing complexity of the Manakov models with larger number of components. It will be worthwhile if analytical and computational predictions here can be verified in experiments in the future. More precisely, rogue wave solutions for the defocusing three-component Manakov system with group velocity mismatch are derived by the Hirota bilinear method. The nonlinear coupling effect is highlighted, namely, the extension of existence regime of rogue waves and the enhancement of amplitude. The main focus of the work is to demonstrate the generation of rogue wave from baseband disturbance.

Recently, the onset of baseband modulation instability has been proven to be equivalent to the existence condition of rogue waves in several systems. In this work, the role of low frequency disturbance in the formation of rogue waves is further consolidated through numerical simulations. By perturbing the plane wave solution by a single mode disturbance with a small frequency, rogue wave modes with configuration similar to the analytical rogue wave solutions can be generated. This idea can be generalized to approximate rogue wave modes in non-integrable systems where most analytical methods fail. The proposed numerical methodology can greatly enrich our knowledge of rogue waves in such systems. Detailed investigations on evolution of rogue waves in general systems of coupled nonlinear Schrödinger equations without any restriction on coefficients would be conducted in the future. Moreover, modified NLS equation or the corresponding systems can be studied [50].

Secondly, the single mode wavy disturbance to a plane wave can generate breathers as well. It is well-known that the formation of breathers is closely related to MI. Depending on the MI spectrum, both symmetric and asymmetric breathers can be generated from a single mode wavy disturbance. For the case where there are multiple unstable bands, the unstable mode can generate multiple breathers which superimpose to form an asymmetric breather. On the other hand, a conventional symmetric breather is generated if there exists only one unstable band.

In conclusions, rogue wave and breather formation are closely related to the nature of the MI spectrum. Through the study of the MI spectrum supplemented with numerical simulations, more intriguing wave dynamics of general non-integrable systems can be revealed in the future. Theoretically, three-component or multi-component systems with variable coefficients and external potential can be further investigated through a similarity transformation [13]. Recently, nonlocal equations have been widely studied due to their PT symmetric property [51]. Many nonlocal evolution equations display remarkable similarities in comparison with intensively studied classical ones, e.g., the nonlocal NLS equation

$$
i A_{z}+A_{t t}+A A^{*}(-t, z) A=0,
$$

which can be analyzed by direct and inverse scattering techniques [51]. Indeed, the nonlocal NLS model (Equation (9)) also possesses an infinite number of conservation laws. Thus total self-induced potential $A(t, z) A^{*}(-t, z)$ over the entire spatial domain is conserved, but not the usual intensity $A A^{*}$. 
This feature may have implications on the size of the "elevation" and "depression" regions of the rogue waves [52]. Naturally the structure of the scattering problem and Painlevé property are slightly different from those of the classical NLS equation. These features and other extensions, e.g., coupled waveguides, higher order dispersion, and discrete models will likely constitute fruitful paths of research in the future.

Acknowledgments: Partial financial support for this project has been provided by the Research Grants Council contracts HKU711713E and HKU17200815.

Author Contributions: Kwok Wing Chow initiated the work. Hiu Ning Chan performed the analysis and simulations. Both authors participated in the interpretation of the results and the writing of the paper.

Conflicts of Interest: The authors declare no conflict of interest.

\section{Appendix A}

To analyze the shape of the rogue wave solution, consider the normalized intensity function of $w$ defined by $F=\frac{|w|^{2}}{\rho^{2}}$. At the stationary point $(0,0)$, the second derivatives are given by $\left.F_{t t}\right|_{(0,0)}=\frac{48 a^{4}\left(b^{2}-a^{2}\right)}{\left(a^{2}+b^{2}\right)^{2}}$ and $\left.\left(F_{z z} F_{t t}-F_{z t}^{2}\right)\right|_{(0,0)}=\frac{256 a^{10}\left(3 a^{2}-b^{2}\right)\left(a^{2}-3 b^{2}\right)}{\left(a^{2}+b^{2}\right)^{4}}$. Similarly, for the evolution of $u$ and $v, b$ is replaced by $b-\delta$ and $b+\delta$ respectively.

From the real part and imaginary part of the dispersion relation Equation (3), note that

$$
\begin{gathered}
{\left[2\left(\delta^{2}+3 \sigma \rho^{2}\right)+3 c\right] d^{2}=c^{3}+2\left(\delta^{2}+3 \sigma \rho^{2}\right) c^{2}+\delta^{4} c+2 \delta^{4} \sigma \rho^{2} \text { and }} \\
d^{2}=3 c^{2}+4\left(\delta^{2}+3 \sigma \rho^{2}\right) c+\delta^{4}
\end{gathered}
$$

where $c=a^{2}-b^{2}$ and $d=2 a b$. It can be deduced that $c$ is negative. Otherwise equating $d^{2}$ in both equations will result in a cubic equation in $c, 8 c^{3}+16\left(\delta^{2}+3 \sigma \rho^{2}\right) c^{2}+\left[2 \delta^{4}+8\left(\delta^{2}+3 \sigma \rho^{2}\right)^{2}\right] c+2 \delta^{6}+$ $4 \delta^{4} \sigma \rho^{2}=0$, which leads to a contradiction since the polynomial is positive if $c$ is positive.

Existence of EDRW with non-zero lower bound

Without loss of generality, consider the case when both $a$ and $b$ are positive. If $a=b$, then it is trivial from the second order derivative test that $|v|$ attains minimum at $(0,0)$. The case when $a>b$ will lead to a contradiction, so it remains to consider the case when $a<b$. From Equation (A1), it can be shown that $\delta^{2}-2 c>\sqrt{c^{2}+d^{2}}$ which implies that $(b+\delta)^{2}-3 a^{2}>2 b \delta>0$. From the second order derivatives, it can be concluded that $v$ is an EDRW when $\Omega_{0}=a+i b$. On the other hand, $u$ is an EDRW in the solution corresponding to the alternative root, $\Omega_{0}=a-i b$. Hence, there must be a component with the configuration of an EDRW.

Moreover, the amplitude of the dark rogue wave has a lower bound of about $0.4 \varrho$. By considering the coefficient of the quadratic term in the dispersion relation, it can be shown that $\delta>\sqrt{2} a$. With $b>a$ and $(b+\delta)^{2}-3 a^{2}>2 b \delta$, it is easy to obtain the bound $|v|>\frac{1}{1+\sqrt{2}} \rho \approx 0.4142 \rho$. In particular, $v$ cannot be a black rogue wave with zero minimum intensity.

Non-existence of eye-shaped bright rogue wave

Since $a^{2}<b^{2},(0,0)$ is either a minimum point or a saddle point. Hence, $w$ cannot be an eye-shaped bright rogue wave. Similarly, $u$ and $v$ cannot be an eye-shaped bright rogue wave. When $a^{2}>(b+\delta)^{2}$, then $a^{2}>b^{2}$ and from the above analysis $3 b^{2}>a^{2}$. It is then obvious that $3(b+\delta)^{2}>a^{2}$ and from the sign of the Hessian, it can be concluded that $(0,0)$ is a saddle point for $|v|$ and $v$ cannot be an eye-shaped bright rogue wave. Furthermore, by the symmetry between $u$ and $v$, it is trivial that $u$ cannot possess such configuration either.

\section{References}

1. Kharif, C.; Pelinovsky, E.; Slunyaev, A. Rogue Waves in the Ocean, 1st ed.; Springer: Berlin, Germany, 2009.

2. Dysthe, K.B.; Krogstad, H.E.; Müller, P. Oceanic rogue waves. Ann. Rev. Fluid Mech. 2008, 40, 287-310. [CrossRef] 
3. Adcock, T.A.A.; Taylor, P.H. The physics of anomalous ('rogue') ocean waves. Rep. Prog. Phys. 2014, 77, 105901. [CrossRef] [PubMed]

4. Cousins, W.; Sapsis, T.P. Quantification and prediction of extreme events in a one-dimensional nonlinear dispersive wave model. Physica D 2014, 280-281, 48-58. [CrossRef]

5. Onorato, M.; Residori, S.; Bortolozzo, U.; Montina, A.; Arecchi, F.T. Rogue waves and their generating mechanisms in different physical contexts. Phys. Rep. 2013, 528, 47-89. [CrossRef]

6. Shemer, L.; Alperovich, L. Peregrine breather revisited. Phys. Fluids 2013, 25, 051701. [CrossRef]

7. Grimshaw, R.H.J.; Tovbis, A. Rogue waves: analytical predictions. Proc. R. Soc. A 2013, 469, 20130094. [CrossRef]

8. Ablowitz, M.J.; Horikis, T.P. Interacting nonlinear wave envelopes and rogue wave formation in deep water. Phys. Fluids 2012, 27, 012107. [CrossRef]

9. Onorato, M.; Residori, S.; Baronio, F. Rogue and Shock Waves in Nonlinear Dispersive Media, 1st ed.; Springer: Basel, Switzerland, 2016.

10. Solli, D.R.; Ropers, C.; Koonath, P.; Jalali, B. Optical rogue waves. Nature 2007, 450, 1054-1058. [CrossRef] [PubMed]

11. Dudley, J.M.; Dias, F.; Erkintalo, M.; Genty, G. Instabilities, breathers and rogue waves in optics. Nat. Photonics 2014, 8, 755-764. [CrossRef]

12. Akhmediev, N.; Kibler, B.; Baronio, F.; Belić, M.; Zhong, W.-P.; Zhang, Y.; Chang, W.; Soto-Crespo, J.M.; Vouzas, P.; Grelu, P.; et al. Roadmap on optical rogue waves and extreme events. J. Opt. 2016, 18, 063001. [CrossRef]

13. Zhong, W.-P.; Belić, M.; Malomed, B.A. Rogue waves in a two-component Manakov system with variable coefficients and an external potential. Phys. Rev. E 2015, 92, 053201. [CrossRef] [PubMed]

14. Akhmediev, N.; Ankiewicz, A.; Soto-Crespo, J.M. Rogue waves and rational solutions of the nonlinear Schrödinger equation. Phys. Rev. E 2009, 80, 026601. [CrossRef] [PubMed]

15. Shrira, V.I.; Geogjaev, V.V. What makes the Peregrine soliton so special as a prototype of freak waves? J. Eng. Math. 2010, 67, 11-22. [CrossRef]

16. Chan, H.N.; Chow, K.W.; Kedziora, D.J.; Grimshaw, R.H.J.; Ding, E. Rogue wave modes for a derivative nonlinear Schrödinger model. Phys. Rev. E 2014, 89, 032914. [CrossRef] [PubMed]

17. Dhar, A.K.; Das, K.P. Fourth-order nonlinear evolution equation for two Stokes wave trains in deep water. Phys. Fluids 1991, 3, 3021-3026. [CrossRef]

18. Gramstad, O.; Trulsen, K. Fourth-order coupled nonlinear Schrödinger equations for gravity waves on deep water. Phys. Fluids 2011, 23, 062102. [CrossRef]

19. Chen, Z.; Segev, M.; Coskun, T.H.; Christodoulides, D.N.; Kivshar, Y.S. Coupled photorefractive spatial-soliton pairs. J. Opt. Soc. Am. B 1997, 14, 3066-3077. [CrossRef]

20. Agrawal, G.P. Nonlinear Fiber Optics, 4th ed.; Academic Press: New York, NY, USA, 2006.

21. Mecozzi, A.; Antonelli, C.; Shtaif, M. Nonlinear propagation in multi-mode fibers in the string coupling regime. Opt. Express 2012, 20, 11673-11678. [CrossRef] [PubMed]

22. Mumtaz, S.; Essiambre, R.; Agrawal, G.P. Nonlinear propagation in multimode and multicore fibers: generalization of the Manakov equations. J. Lightwave Technol. 2013, 31, 398-406. [CrossRef]

23. Guasoni, M. Generalized modulational instability in multimode fibers: wideband multimode parametric amplification. Phys. Rev. A 2015, 92, 033849. [CrossRef]

24. Wang, D.S.; Shi, Y.R.; Chow, K.W.; Yu, Z.X.; Li, X.G. Matter-wave solitons in a spin-1 Bose-Einstein condensate with time-modulated external potential and scattering lengths. Eur. Phys. J. D 2013, 67, 242. [CrossRef]

25. Baronio, F.; Degasperis, A.; Conforti, M.; Wabnitz, S. Solutions of the vector nonlinear Schrödinger equations: evidence for deterministic rogue waves. Phys. Rev. Lett. 2012, 109, 044102. [CrossRef] [PubMed]

26. Ling, L.; Guo, B.; Zhao, L.C. High-order rogue waves in vector nonlinear Schrödinger equations. Phys. Rev. E 2014, 89, 041201(R). [CrossRef] [PubMed]

27. He, J.; Guo, L.; Zhang, Y.; Chabchoub, A. Theoretical and experimental evidence of non-symmetric doubly localized rogue waves. Proc. R. Soc. A 2014, 470, 20140318. [CrossRef] [PubMed]

28. Degasperis, A.; Lombardo, S. Rational solitons of wave resonant-interaction models. Phys. Rev. E 2013, 88, 052914. [CrossRef] [PubMed]

29. Baronio, F.; Conforti, M.; Degasperis, A.; Lombardo, S.; Onorato, M.; Wabnitz, S. Vector rogue waves and baseband modulation instability in the defocusing regime. Phys. Rev. Lett. 2014, 113, 034101. [CrossRef] [PubMed] 
30. Chen, S.; Soto-Crespo, J.M.; Grelu, P. Dark three-sister rogue waves in normally dispersive optical fibers with random birefringence. Opt. Express 2014, 22, 27632-27642. [CrossRef] [PubMed]

31. Li, J.H.; Chan, H.N.; Chiang, K.S.; Chow, K.W. Breathers and 'black' rogue waves of coupled nonlinear Schrödinger equations with dispersion and nonlinearity of opposite signs. Commun. Nonlinear Sci. Numer. Simulat. 2015, 28, 28-38. [CrossRef]

32. Frisquet, B.; Kibler, B.; Fatome, J.; Morin, P.; Baronio, F.; Conforti, M.; Millot, G.; Wabnitz, S. Polarization modulation instability in a Manakov fiber system. Phys. Rev. A 2015, 92, 053854. [CrossRef]

33. Frisquet, B.; Kibler, B.; Morin, P.; Baronio, F.; Conforti, M.; Millot, G.; Wabnitz, S. Optical dark rogue wave. Sci. Rep. 2016, 6, 20785. [CrossRef] [PubMed]

34. Zhao, L.C.; Liu, J. Rogue-wave solutions of a three-component coupled nonlinear Schrödinger equation. Phys. Rev. E 2013, 87, 013201. [CrossRef] [PubMed]

35. Liu, C.; Yang, Z.-Y.; Zhao, L.-C.; Yang, W.-L. Vector breathers and the inelastic interaction in a three-mode nonlinear optical fiber. Phys. Rev. A 2014, 89, 055803. [CrossRef]

36. $\mathrm{Xu}, \mathrm{T}$;; Chen, Y. Localized waves in three-component coupled nonlinear Schrödinger equation. Chin. Phys. $B$ 2016, 25, 090201. [CrossRef]

37. Hirota, R. The Direct Method in Soliton Theory, 1st ed.; Cambridge University Press: Cambridge, UK, 2004.

38. Chan, H.N.; Malomed, B.A.; Chow, K.W.; Ding, E. Rogue waves for a system of coupled derivative nonlinear Schrödinger equations. Phys. Rev. E 2016, 93, 012217. [CrossRef] [PubMed]

39. Chan, H.N.; Chow, K.W. Rogue waves for an alternative system of coupled Hirota equations: structural robustness and modulation instabilities. Stud. Appl. Math. 2017. [CrossRef]

40. Chen, S.; Soto-Crespo, J.M.; Grelu, P. Coexisting rogue waves within the $(2+1)$-component long-wave-short-wave resonance. Phys. Rev. E 2014, 90, 033203. [CrossRef] [PubMed]

41. Chan, H.N.; Ding, E.; Kedziora, D.J.; Grimshaw, R.H.J.; Chow, K.W. Rogue waves for a long wave-short wave resonance model with multiple short waves. Nonlinear Dyn. 2016, 85, 2827-2841. [CrossRef]

42. Baronio, F.; Chen, S.; Grelu, P.; Wabnitz, S.; Conforti, M. Baseband modulation instability as the origin of rogue waves. Phys. Rev. A 2015, 91, 033804. [CrossRef]

43. Yang, J. Nonlinear Waves in Integrable and Nonintegrable Systems, 1st ed.; SIAM: Philadelphia, PA, USA, 2010.

44. Wang, L.; Zhang, L.-L.; Zhu, Y.-J.; Qi, F.-H.; Wang, P.; Guo, R.; Li, M. Modulational instability, nonautonomous characteristics and semirational solutions for the coupled nonlinear Schrödinger equations in inhomogeneous fibers. Commun. Nonlinear Sci. Numer. Simul. 2016, 40, 216-237. [CrossRef]

45. Tiofack, C.G.L.; Coulibaly, S.; Taki, M.; De Bièvre, S.; Dujardin, G. Comb generation using multiple compression points of Peregrine rogue waves in periodically modulated nonlinear Schrödinger equations. Phys. Rev. A 2015, 92, 043837. [CrossRef]

46. Baronio, F. Akhmediev breathers and Peregrine solitary waves in a quadratic medium. Opt. Lett. 2017, 42, 1756-1759. [CrossRef] [PubMed]

47. Manikandan, K.; Senthilvelan, M.; Kraenkel, R.A. On the characterization of vector rogue waves in two-dimensional two coupled nonlinear Schrödinger equations with distributed coefficients. Eur. Phys. J. B 2016, 89, 218. [CrossRef]

48. Tan, Z.; Tian, B.; Jiang, Y.; Wang, P.; Li, M. Dynamics of the Manakov solitons in biased guest-host photorefractive polymer. Commun. Theor. Phys. 2013, 60, 150-158. [CrossRef]

49. Tsitsas, N.L.; Lakhtakia, A.; Frantzeskakis, D.J. Vector solitons in nonlinear isotropic chiral metamaterials. J. Phys. A Math. Theor. 2011, 44, 435203. [CrossRef]

50. Boscolo, S.; Peng, J.; Finot, C. Design and applications of in-cavity pulse shaping by spectral sculpturing in mode-locked fibre lasers. Appl. Sci. 2015, 5, 1379-1398. [CrossRef]

51. Ablowitz, M.J.; Musslimani, Z.H. Integrable nonlocal nonlinear Schrödinger equation. Phys. Rev. Lett. 2013, 110, 064105. [CrossRef] [PubMed]

52. Gupta, S.K.; Sarma, A.K. Peregrine rogue wave dynamics in the continuous nonlinear Schrödinger system with parity-time symmetric Kerr nonlinearity. Commun. Nonlinear Sci. Numer. Simulat. 2016, 36, 141-147. [CrossRef]

(C) 2017 by the authors. Licensee MDPI, Basel, Switzerland. This article is an open access article distributed under the terms and conditions of the Creative Commons Attribution (CC BY) license (http://creativecommons.org/licenses/by/4.0/). 